\title{
Mathematical structure of entanglement catalysis
}

\author{
Sumit Daftuar* \\ Institute for Quantum Information, California Institute of Technology, Pasadena, California 91125 \\ Matthew Klimesh ${ }^{\dagger}$ \\ Jet Propulsion Laboratory, California Institute of Technology, Pasadena, California 91109
}

(Received 24 April 2001; published 18 September 2001)

\begin{abstract}
The majorization relation has been shown to be useful in classifying which transformations of jointly held quantum states are possible using local operations and classical communication. In some cases, a direct transformation between two states is not possible, but it becomes possible in the presence of another state (known as a catalyst); this situation is described mathematically by the trumping relation, an extension of majorization. The structure of the trumping relation is not nearly as well understood as that of majorization. We give an introduction to this subject and derive some results. Most notably, we show that the dimension of the required catalyst is, in general, unbounded; there is no integer $k$ such that it suffices to consider catalysts of dimension $k$ or less in determining which states can be catalyzed into a given state. We also show that almost all bipartite entangled states are potentially useful as catalysts.
\end{abstract}

DOI: 10.1103/PhysRevA.64.042314

PACS number(s): 03.67.-a

\section{INTRODUCTION}

The study of quantum entanglement has received considerable attention in recent years, with numerous remarkable applications including quantum cryptography [1,2], quantum teleportation [3], and superdense coding [4]. Entanglement seems to be the essential element of such applications, and as a result it has come to be viewed as a fundamental resource that allows one to perform certain information-processing tasks. As with any physical resource, one wishes to measure how much entanglement is present in a given system, and to determine under what conditions it is possible to convert one form of entanglement to another. The problem of how to quantify and classify entanglement is one of the basic questions in the rapidly growing science of quantum information theory $[5,6]$.

A significant advance in understanding entanglement was made by Nielsen, who showed [7] that the structure of the bipartite entangled states is related to the linear-algebraic theory of majorization $[8,9]$. We give an introduction to this subject here. Suppose that $x=\left(x_{1}, \ldots, x_{d}\right)$ and $y$ $=\left(y_{1}, \ldots, y_{d}\right)$ are $d$-dimensional probability vectors; in other words, their components are non-negative and sum to unity. We let $x \downarrow$ denote the $d$-dimensional vector obtained by arranging the components of $x$ in nonincreasing order: $x^{\downarrow}$ $=\left(x_{1}^{\downarrow}, \ldots, x_{d}^{\downarrow}\right)$, where $x_{1}^{\downarrow} \geqslant x_{2}^{\downarrow} \geqslant \cdots \geqslant x_{d}^{\downarrow}$. Then we say that $x$ is majorized by $y$, written $x<y$, if the following relations hold:

$$
\sum_{i=1}^{l} x_{i}^{\downarrow} \leqslant \sum_{i=1}^{l} y_{i}^{\downarrow} \quad(1 \leqslant l<d) .
$$

(In fact, the theory of majorization is not limited to probability vectors. The majorization relation can be defined as

\footnotetext{
*Electronic address: daftuar@its.caltech.edu

${ }^{\dagger}$ Electronic address: klimesh@shannon.jpl.nasa.gov
}

above for any real vectors $x$ and $y$, if we include the additional restriction that $\sum_{i=1}^{d} x_{i}=\sum_{i=1}^{d} y_{i}$, which is automatically satisfied for probability vectors. For our applications to the study of entanglement, however, $x$ and $y$ will always be probability vectors, and we will make this assumption throughout.)

Intuitively, if $x$ and $y$ are probability vectors such that $x$ $<y$, then $x$ describes an unambiguously more random distribution than does $y$. For example, in $R^{2}$, we have that $(0.5,0.5)<(0.8,0.2)$. In fact, $(0.5,0.5)$ is majorized by every vector in $R^{2}$ whose components sum to unity.

The majorization relation defines a partial order on $d$-dimensional real vectors, where $x<y$ and $y<x$, if and only if $x^{\downarrow}=y^{\downarrow}$. To see that majorization is not a complete relation, consider for instance $x=(0.5,0.25,0.25)$ and $y$ $=(0.4,0.4,0.2)$; then $x \nless y$ and $y \nless x$.

We are now ready to state Nielsen's theorem [7].

Theorem 1. Suppose Alice and Bob are in joint possession of a bipartite entangled quantum state $|\psi\rangle$ which they wish to transform into another bipartite entangled state $|\phi\rangle$ using only local operations and classical communication (LOCC). Let $|\psi\rangle=\sum_{i=1}^{d} \sqrt{\alpha_{i}}\left|i_{A}\right\rangle\left|i_{B}\right\rangle$ be a Schmidt decomposition of $|\psi\rangle$, and let $|\phi\rangle=\sum_{i=1}^{d} \sqrt{\beta_{i}}\left|i_{A}^{\prime}\right\rangle\left|i_{B}^{\prime}\right\rangle$ be a Schmidt decomposition of $|\phi\rangle$. Then $|\psi\rangle$ can be converted to $|\phi\rangle$ by LOCC, if and only if the vector $\alpha=\left(\alpha_{1}, \ldots, \alpha_{d}\right)$ is majorized by $\beta=\left(\beta_{1}, \ldots, \beta_{d}\right)$.

Nielsen's theorem defines a partial order on the entangled bipartite pure states. If state $|\psi\rangle$ has $x$ as its vector of Schmidt coefficients, and $|\phi\rangle$ has $y$ as its vector of Schmidt coefficients, then we can transform $|\psi\rangle$ to $|\phi\rangle$ using LOCC, if and only if $x<y$. Because our ability to transform one state to another depends only on their Schmidt coefficients, and not on the bases, we shall abuse nomenclature and refer to any vector of Schmidt coefficients as a "state."

The above characterization of when one entangled state can be transformed to another is particularly helpful because the structure of the majorization relation is relatively well understood. For example, the following results are well known [8]. 
Theorem 2. Let $x, y \in R^{d}$. Then (a) The following are equivalent:

(i) $x<y$.

(ii) $\sum_{i=1}^{d} x_{i}=\sum_{i=1}^{d} y_{i}$ and for all $l \in\{2, \ldots, d\}, \quad \sum_{i=l}^{d} x_{i}^{\downarrow}$ $\geqslant \sum_{i=l}^{d} y_{i}^{\downarrow}$.

(iii) $x=D y$ for some doubly stochastic $d \times d$ matrix $D$.

(iv) For every real number $t, \sum_{i=1}^{d}\left|x_{i}-t\right| \leqslant \sum_{i=1}^{d}\left|y_{i}-t\right|$.

(b) Let $S(y)=\left\{x \in R^{d} \mid x<y\right\}$. Then $S(y)$ is a convex set whose extreme points are the elements of the set $\left\{P_{y} \mid P\right.$ is a $d \times d$ permutation matrix $\}$.

Jonathan and Plenio have extended Nielsen's result by describing a phenomenon known as entanglement catalysis [10]. Suppose that $x=(0.4,0.4,0.1,0.1)$ and $y$ $=(0.5,0.25,0.25,0)$. Then $x \nless y$. Now let $z=(0.6,0.4)$. Then we have $x \otimes z<y \otimes z$. In other words, if Alice and Bob start only with state $x$ (by which we mean a jointly entangled quantum state whose Schmidt coefficients are the components of $x$ ), they cannot transform it into state $y$ using LOCC. But if they also have state $z$ available, then they can turn $x$ $\otimes z$ into $y \otimes z$. So they can "borrow" $z$, use it to help turn $x$ into $y$, and "return" it after performing the transformation. We say that $z$ is a catalyst for the transformation.

The phenomenon of catalysis illustrates that entanglement itself can be used as a resource to help perform transformations of entangled states. One naturally wishes to know when this is possible: given $x$ and $y$, can we determine whether $x$ can be transformed to $y$ using LOCC in the presence of a catalyst? This is equivalent to asking whether there is a probability vector $z$ such that $x \otimes z<y \otimes z$.

We will adopt the terminology and notation introduced by Nielsen [9] and say that $x$ is trumped by $y$, written $x<_{T} y$, if there exists a catalyst $z$ (of any dimension) such that $x \otimes z$ $<y \otimes z$. For any given $y$, let $T(y)$ denote the set of all $x$ such that $x$ is trumped by $y$; and for any $y$ and $z$, let $T(y, z)$ be the set of all $x$ such that $x \otimes z<y \otimes z$. In addition, we introduce the following notation: for any $d$-dimensional probability vector $y$ and any positive integer $k$, let $T_{k}(y)=\{x \mid \exists$ a $k$-dimensional probability vector $z$ such that $x \otimes z<y \otimes z\}$.

Our results will rely heavily on the fact that the trumping relation involves vectors with all non-negative components. Note that this is quite different from the situation with majorization, in which most results extend easily to vectors containing negative components.

The following facts are known about the trumping relation. The first three are straightforward from the definitions; the others have been proven elsewhere [10,9].

Theorem 3. Let $x$ and $y$ be $d$-dimensional probability vectors, let $z$ be a probability vector (of any dimension), and let $S(y), T(y)$, and $T_{k}(y)$ be defined as above. Then

(a) $x<y \Rightarrow x \otimes z<y \otimes z$.

(b) $S(y) \subseteq T(y)$.

(c) $T(y)=\cup_{k=1}^{\infty} T_{k}(y)$.

(d) $T(y)$ is a convex set.

(e) If $x<_{T} y$ and $y<_{T} x$, then $x \downarrow=y^{\downarrow}$.

(f) If $x<_{T} y$, then $x_{1}^{\downarrow} \leqslant y_{1}^{\downarrow}$ and $x_{d}^{\downarrow} \geqslant y_{d}^{\downarrow}$

In contrast to the situation with the majorization relation, the mathematical structure of the trumping relation is not well understood. One desires a necessary and sufficient con- dition for determining whether $x<_{T} y$ [or alternately, to determine the elements of the set $T(y)$ for any given $y$ ]. Characterizing the trumping relation in this way would help us to better understand the structure of the bipartite entangled states. However, such a characterization is not yet known.

In examining the trumping relation, many questions naturally arise. For instance, if $y=(1 / d, \ldots, 1 / d)$, the trumping condition is (trivially) the same as the majorization condition: $x<y$, if and only if $x<_{T} y$. One wishes to know for which $y$ this is the case. One also desires to know whether catalysts of arbitrarily high dimension need be considered, in the following sense: given $y$, is it possible to find $k$ such that $T_{k}(y)=T(y)$ ? These questions are among those answered in this paper.

\section{A KEY LEMMA}

The following lemma and its corollary will be useful to us in proving additional results, and are also interesting in their own right:

Lemma 4. Let $x=\left(x_{1}, \ldots, x_{d}\right)$ and $y=\left(y_{1}, \ldots, y_{d}\right)$ be $d$-dimensional probability vectors, whose components we assume to be arranged in nonincreasing order: $x_{1} \geqslant x_{2} \geqslant \cdots$ $\geqslant x_{d}$, and similarly for $y$. Suppose that $x<y, y_{1}>x_{1}$, and $y_{d}<x_{d}$. Then $x$ is in the interior of $T(y)$.

Note that when we say $x$ is in the interior of $T(y)$ we mean the interior relative to the space of $d$-dimensional probability vectors; that is, for any $x$ there must exist an $\epsilon$ such that if $x^{\prime}$ is a probability vector for which $\left\|x^{\prime}-x\right\|<\epsilon$ (in the Euclidean norm, for instance), then $x^{\prime} \in T(y)$.

We remark that the conclusion is obvious if $x$ is in the interior of $S(y)$; the important fact is that the result holds when $x$ is on the boundary of $S(y)$.

Proof. Note that $x_{d}>0$. Pick an $\alpha$ satisfying $\alpha<1, \alpha$ $>x_{1} / y_{1}$, and $\alpha>y_{d} / x_{d}$. Let $k$ be an integer for which $x_{1} \alpha^{k-1}<x_{d}$. Now let $z$ be the $k$-dimensional vector

$$
z=\left(1, \alpha, \ldots, \alpha^{k-1}\right) .
$$

(Of course $z$ is not a probability vector, but it can easily be normalized. For convenience in the proof, we neglect the normalization.)

We will show that $x$ is in the interior of $T(y, z)$. Since $T(y, z) \subset T(y)$, this will establish the result.

Let $(y \otimes z)_{i}^{\downarrow}$ denote the $i$ th component of $y \otimes z$ when its components are arranged in nonincreasing order. We will show that for $1 \leqslant l \leqslant d k-1$,

$$
\sum_{i=1}^{l}(x \otimes z)_{i}^{\downarrow}<\sum_{i=1}^{l}(y \otimes z)_{i}^{\downarrow} .
$$

Note that since $x \otimes z$ must be majorized by $y \otimes z$, we already know that Eq. (1) must hold for $0 \leqslant l \leqslant d k$ if " $<$ " is replaced by " $\leqslant$ " (and this fact is used later in the proof). Showing that Eq. (1) holds for $1 \leqslant l \leqslant d k-1$ will complete the proof since it is then clear that any sufficiently small perturbations to $x$ (within the probability space) will not cause Eq. (1) to be violated for any $1 \leqslant l \leqslant d k-1$. 
For the remainder of the proof we fix $l$ as an arbitrary integer satisfying $1 \leqslant l \leqslant d k-1$. Consider the terms that the left-hand sum of Eq. (1) will contain. For $1 \leqslant i \leqslant d$, let $r_{i}$ denote the number of these terms that are of the form $x_{i} \alpha^{j}$, with $0 \leqslant j<k$. (In case of repeated values of components of $x \otimes z$, we regard terms with smaller $i$ to be included in the sum first.) Note that these $r_{i}$ terms must be $x_{i}, x_{i} \alpha, \ldots, x_{i} \alpha^{r_{i}-1}$, since these are the largest of this form. The sum (which we denote by $s_{x}$ ) can thus be written

$$
s_{x}=\sum_{i=1}^{d} \sum_{j=0}^{r_{i}-1} x_{i} \alpha^{j}
$$

Note that $0 \leqslant r_{i} \leqslant k$ and in addition $r_{1}>0$ and $r_{d}<k$.

Consider the sum

$$
s_{y}=\sum_{i=1}^{d} \sum_{j=0}^{r_{i}-1} y_{i} \alpha^{j}
$$

The terms of this sum may or may not be the $l$ largest components of $y \otimes z$, but if $s_{x}<s_{y}$ then we are done because $s_{y}$ is less than or equal to the right-hand sum in Eq. (1). The fact that $x<y$ implies that $s_{x} \leqslant s_{y}$; this follows from comparing the terms in the sums with a fixed $j$. Thus we need only consider the case $s_{x}=s_{y}$.

Let $m_{y}$ be the minimum of the terms included in the sum in Eq. (3) and let $M_{y}$ be the maximum of those components of $y \otimes z$ that are not included in this sum. Define $m_{x}$ and $M_{x}$ analogously. If $M_{y}>m_{y}$, we are done, since the largest term not in the sum in Eq. (3) can be swapped with the smallest one in the sum, implying Eq. (1). We assume that $M_{y} \leqslant m_{y}$ and show that a contradiction will follow.

There are two cases to consider. We first consider the case where $r_{1}<k$ (that is, $r_{1} \neq k$ ). Note that our current assumptions (including $M_{y} \leqslant m_{y}$ ) imply $m_{y} \leqslant m_{x}$, since otherwise we would have

$$
\sum_{i=1}^{l-1}(x \otimes z)_{i}^{\downarrow}>\sum_{i=1}^{l-1}(y \otimes z)_{i}^{\downarrow} .
$$

It follows that

$$
m_{y} \leqslant m_{x} \leqslant x_{1} \alpha^{r_{1}-1}<y_{1} \alpha^{r_{1}} \leqslant M_{y},
$$

where we have used one of our requirements on $\alpha$ as well as the facts that $x_{1} \alpha^{r_{1}-1}$ is in the sum in Eq. (2) and $y_{1} \alpha^{r_{1}}$ is not in the sum in Eq. (3). But Eq. (4) contradicts our assumption that $M_{y} \leqslant m_{y}$, so the first case is complete.

In the other case $r_{1}=k$, so $m_{x} \leqslant x_{1} \alpha^{k-1}$. But $x_{1} \alpha^{k-1}$ $<x_{d}$ by our choice of $k$, so we must have $r_{d}>0$. Our assumptions imply that $M_{y} \geqslant M_{x}$, since otherwise we would have

$$
\sum_{i=1}^{l+1}(x \otimes z)_{i}^{\downarrow}>\sum_{i=1}^{l+1}(y \otimes z)_{i}^{\downarrow} .
$$

Therefore,

$$
M_{y} \geqslant M_{x} \geqslant x_{d} \alpha^{r_{d}>y_{d}} \alpha^{r_{d}-1} \geqslant m_{y}
$$

by reasoning similar to that yielding Eq. (4). Again our assumption that $M_{y} \leqslant m_{y}$ is contradicted. Thus the proof is complete.

Corollary 5. Suppose $x$ and $y$ are $d$-dimensional probability vectors, with components arranged in nonincreasing order, such that $x<_{T} y$ and $y_{1}>x_{1}$ and $y_{d}<x_{d}$. Then $x$ is in the interior of $T(y)$.

Proof. By definition there exists a $z$ such that $x \otimes z<y$ $\otimes z$. Since $y_{1}>x_{1}$ and $y_{d}<x_{d}$ we must have $(x \otimes z)_{1}^{\downarrow}<(y$ $\otimes z)_{1}^{\downarrow}$ and $(x \otimes z)_{d k}^{\downarrow}>(y \otimes z)_{d k}^{\downarrow}$, where $k$ is the dimension of $z$.

We can thus apply Lemma 4 and conclude that $x \otimes z$ is in the interior of $T(y \otimes z)$. Since $x \mapsto x \otimes z$ is a continuous function, it follows that $x$ is in the interior of $\{x \mid x \otimes z \in T(y$ $\otimes z)\}$. But $\{x \mid x \otimes z \in T(y \otimes z)\}=T(y)$, so we are done.

\section{WHEN IS CATALYSIS USEFUL?}

If $T(y)=S(y)$, then catalysis is of no help in producing the state $y$. This is obviously the case when $y=(1,0, \ldots, 0)$, for then all vectors in $R^{d}$ are in both $S(y)$ and $T(y)$. Jonathan and Plenio have shown [10] that if $d \leqslant 3$ then $x<_{T} y \Rightarrow x$ $<y$; in other words, $S(y)=T(y)$ if $y$ is at most three dimensional. The following theorem shows that for almost all vectors $y$ of four or more dimensions, $S(y) \neq T(y)$.

Theorem 6. Let $y=\left(y_{1}, \ldots, y_{d}\right)$ be a $d$-dimensional probability vector whose components are in nonincreasing order. Then $T(y) \neq S(y)$ if and only if $y_{1} \neq y_{l}$ and $y_{m} \neq y_{d}$ for some $l, m$ with $1<l<m<d$.

This theorem says that $S(y) \neq T(y)$, if and only if $y$ has at least two components that are distinct from both its smallest and largest components.

Proof. Suppose that there exist such $l$ and $m$. Let $d_{1}$ be the number of components of $y$ equal to $y_{1}$, and let $d_{2}$ be the number of components of $y$ equal to $y_{d}$. Then $d_{1}+d_{2}+2$ $\leqslant d$. Let $x$ be the $d$-dimensional vector whose first $d_{1}+1$ components are each equal to the average of the first $d_{1}+1$ components of $y$, whose last $d_{2}+1$ components are each equal to the average of the last $d_{2}+1$ components of $y$ whose remaining components are equal to the corresponding components of $y$. Then it is easily checked that $x<y$. In fact, $x$ is on the boundary of $S(y)$ since $\sum_{i=1}^{d_{1}+1} x_{i}=\sum_{i=1}^{d_{1}+1} y_{i}$. However, by Corollary 5, $x$ is in the interior of $T(y)$; thus $S(y)$ $\neq T(y)$.

Conversely, assume that there are no $l, m$ such that $l<m$, $y_{1} \neq y_{l}$, and $y_{m} \neq y_{d}$. Again let $d_{1}$ be the number of components of $y$ equal to $y_{1}$, and $d_{2}$ the number of components equal to $y_{d}$. Let $x \in T(y)$ and assume the components of $x$ are arranged in decreasing order. Then $x_{1} \leqslant y_{1}$, so $\sum_{i=1}^{j} x_{i}$ $\leqslant \sum_{i=1}^{j} y_{i}$ for $j \in\left\{1, \ldots, d_{1}\right\}$. Also $x_{d} \geqslant y_{d}$, so $\sum_{i=j+1}^{d} x_{i}$ $\geqslant \sum_{i=j+1}^{d} y_{i}$, and therefore $\sum_{i=1}^{j} x_{i} \leqslant \sum_{i=1}^{j} y_{i}$, for $j \in\{d$ $\left.-d_{2}, \ldots, d-1\right\}$. But our assumptions imply that $d_{1}+d_{2}+1$ $\geqslant d$, so in fact $\sum_{i=1}^{j} x_{i} \leqslant \sum_{i=1}^{j} y_{i}$ for all $j \in\{1, \ldots, d-1\}$, and so $x<y$. Thus in this case $S(y)=T(y)$.

In applying this theorem, it should be noted that the dimension of $y$ is somewhat arbitrary, as one can append zeroes to the vector $y$ and thereby increase its dimension without changing the underlying quantum state. If the nonzero 
components of $y$ take on exactly two distinct values, and at least two components are equal to the smaller of these values, then appending zeroes will result in a vector $y^{\prime}$ such that $S\left(y^{\prime}\right) \neq T\left(y^{\prime}\right)$, although $S(y)=T(y)$. The reason for this phenomenon is that we only consider vectors $x$ with the same dimension as that of $y$; by increasing the dimension of $y$, we increase the allowed choices for $x$ as well. Thus, the dimension of the initial states $x$ under consideration may determine whether $S(y)=T(y)$.

\section{CATALYSTS OF ARBITRARILY HIGH DIMENSION MUST BE CONSIDERED}

We will now show that for most $y$, there is no $k$ such that $T_{k}(y)=T(y)$. In other words, there is no limit to the dimension of the catalysts that must be considered, in trying to determine which vectors are trumped by a given vector $y$. Our proof will proceed as follows: First we will show that $T_{k}(y)$ is a closed set for any $k$ and all $y$, and then we will show that $T(y)$ is in general not closed. It follows that $T_{k}(y) \neq T(y)$.

The results of the previous section, and of this section, give a precise characterization of when $S(y)=T(y)$, and when there exists a $k$ such that $T_{k}(y)=T(y)$. While it is clear that the former situation implies the latter, it turns out that the converse is true as well.

Theorem 7. $T_{k}(y)$ is closed.

Proof. For a given $d$-dimensional probability vector $y$, let

$$
h(x, z)=\max _{1 \leqslant j<d k} \sum_{i=1}^{j}\left[(x \otimes z)_{i}^{\downarrow}-(y \otimes z)_{i}^{\downarrow}\right],
$$

where $x$ and $z$ are probability vectors of $d$ and $k$ dimensions, respectively. Observe that $h$ is a composition of continuous functions (including the maximum of a finite set of expressions, and the function $x \mapsto x^{\downarrow}$ ) and so is continuous in $x$ and $z$.

Let

$$
f(x)=\min _{z} h(x, z),
$$

where the minimum is over all $k$-dimensional probability vectors $z$; this minimum exists since $h(x, z)$ is continuous in $z$ and the minimization is over a compact set. Observe that $x \in T_{k}(y)$, if and only if $f(x) \leqslant 0$.

Suppose now that $x \notin T_{k}(y)$. Then $f(x)>\epsilon$ for some $\epsilon$ $>0$. Let $x^{\prime}$ be given with $\left\|x-x^{\prime}\right\|<\epsilon / d$. Let $z$ be an arbitrary $k$-dimensional probability vector, let $j_{0}$ be a maximizing value of $j$ in $h(x, z)$, and $\pi$ be a permutation for which ( $x$ $\otimes z)_{i}^{\downarrow}=(x \otimes z)_{\pi(i)}$ for each $i$. Let $v$ be the $d$-dimensional vector $(\epsilon / d, \ldots, \epsilon / d)$ and note that $x_{i}^{\prime}>x_{i}-v_{i}$ for each $i$. We then have

$$
\begin{aligned}
h\left(x^{\prime}, z\right)-h(x, z) & \geqslant \sum_{i=1}^{j_{0}}\left[\left(x^{\prime} \otimes z\right)_{i}^{\downarrow}-(x \otimes z)_{i}^{\downarrow}\right] \\
& \geqslant \sum_{i=1}^{j_{0}}\left[\left(x^{\prime} \otimes z\right)_{\pi(i)}-(x \otimes z)_{\pi(i)}\right]
\end{aligned}
$$

$$
\begin{aligned}
& >\sum_{i=1}^{j_{0}}\left\{[(x-v) \otimes z]_{\pi(i)}-(x \otimes z)_{\pi(i)}\right\} \\
& =-\sum_{i=1}^{j_{0}}(v \otimes z)_{\pi(i)} \\
& \geqslant-\sum_{i=1}^{d k}(v \otimes z)_{\pi(i)} \\
& =-\epsilon
\end{aligned}
$$

Therefore $h\left(x^{\prime}, z\right)>0$ for all $z$, so $f\left(x^{\prime}\right)>0$. We thus see that $x^{\prime} \notin T_{k}(y)$ for $x^{\prime}$ in a neighborhood of $x$. Therefore $T_{k}^{c}(y)$ is open, so $T_{k}(y)$ is closed.

Theorem 8. Let $y=\left(y_{1}, \ldots, y_{d}\right)$ be a $d$-dimensional probability vector, with components in nonincreasing order, such that $T(y) \neq S(y)$. Then for all $k, T_{k}(y) \neq T(y)$.

Proof. By Theorem 6, the hypothesis is equivalent to the existence of $l, m$ such that $1<l<m<d, y_{1}>y_{l}, y_{m}>y_{d}$. For convenience, we redefine $l$ to be the index of the first component of $y$ that is not equal to $y_{1}$, and $m$ to be the index of the last component of $y$ that is not equal to $y_{d}$; clearly we still have $l<m$. Let $\Delta=\min \left\{y_{1}-y_{l}, y_{m}-y_{d}\right\}$ and let $x$ be the $d$-dimensional vector given by $x_{l}=y_{l}+\Delta, x_{m}=y_{m}-\Delta$, and $x_{i}=y_{i}$ for $i \notin\{l, m\}$. It is easily checked that $y<x$ but $x$ $\nless y$, therefore $x \kappa_{T} y$. Let $w=(1 / d, \ldots, 1 / d)$ and note that $w$ $\in S(y)$.

Suppose $T(y)$ is closed. Since $T(y)$ is convex, the set $\{t$ $\in[0,1] \mid t x+(1-t) w \in T(y)\}$ is a closed interval not containing 1 , say $\left[0, t_{0}\right]$. So $T(y)$ contains $t_{0} x+\left(1-t_{0}\right) w$ as a boundary point. But $t_{0} x+\left(1-t_{0}\right) w$ satisfies the hypotheses of Corollary 5 and is thus an interior point of $T(y)$. This is a contradiction, so $T(y)$ cannot be closed. As Theorem 7 says that each $T_{k}(y)$ is closed, we must have $T_{k}(y) \neq T(y)$.

So whenever catalysis is useful in producing $y$ [i.e., $S(y) \neq T(y)$ ], catalysts of arbitrarily high dimension must be considered. In other words, when $S(y) \neq T(y)$, then for any $k$ there is a $k^{\prime}>k$ such that $T_{k}(y)$ is a strict subset of $T_{k^{\prime}}(y)$. However, we do not know whether increasing the catalyst dimension by one will necessarily given an improvement. That is, it is unknown whether there is any vector $y$ and $k$ $\geqslant 1$ such that $S(y) \neq T(y)$ but $T_{k}(y)=T_{k+1}(y)$.

\section{WHICH STATES CAN BE CATALYSTS?}

Another interesting question is that of which states are potentially useful as catalysts. If a vector $z$ is uniform, meaning that its nonzero components are all identical, then it is easily seen that $z$ is not capable of acting as a catalyst; if $x$ $\otimes z<y \otimes z$, then $x<y$ so $z$ served no use as a catalyst. In Ref. [9] Nielsen conjectured that all nonuniform vectors are potentially useful as catalysts. In this section, we show that this conjecture is true.

Before we proceed, let us consider the implications of this conjecture. We know already that a uniform $z$ cannot act as a catalyst. A uniform $z$ with $k$ nonzero components corresponds to a maximally entangled quantum state of Schmidt 
number $k$; if $k=1$ then the state is unentangled. So we have the following situation: if $z$ is a maximally entangled state, then $z$ cannot be used as a catalyst, but for any other entangled state $z$, the conjecture says that $z$ can serve as a catalyst. In using entanglement as a resource, it is possible to have too much as well as too little.

Theorem 9. Let $z=\left(z_{1}, \ldots, z_{k}\right)$ be a nonuniform probability vector. Then there exist probability vectors $x, y \in R^{4}$ such that $x \otimes z<y \otimes z$, but $x \nless y$.

Proof. We may assume without loss of generality that $z_{1}$ $\geqslant z_{2} \geqslant \cdots \geqslant z_{k}>0$. Define $\alpha$ and $\beta$ by the relations

$$
\frac{z_{1}}{z_{k}}=\frac{\alpha}{\beta}
$$

and

$$
\alpha+\beta=1
$$

By nonuniformity of $z, \alpha>\beta$.

Let $x_{1}=x_{2}=\frac{1}{2} \alpha+\frac{1}{4} \beta$, and $x_{3}=x_{4}=\frac{1}{4} \beta$. Let $y_{1}=\alpha$, let $y_{2}=y_{3}=\frac{1}{2} \beta$, and let $y_{4}=0$. Let $x=\left(x_{1}, x_{2}, x_{3}, x_{4}\right)$ and $y$ $=\left(y_{1}, y_{2}, y_{3}, y_{4}\right)$. Note that $x<y$, so obviously $x \otimes z<y$ $\otimes z$. Our goal is to show that all the majorization inequalities between $x \otimes z$ and $y \otimes z$ are strict; in other words, for all $l$ $\in\{1,2, \ldots, 4 k-1\}$,

$$
\sum_{i=1}^{l}(x \otimes z)_{i}^{\downarrow}<\sum_{i=1}^{l}(y \otimes z)_{i}^{\downarrow} .
$$

We will show first that the inequalities are strict when $l$ is even; so for now, assume that $l$ is even. There are five cases to consider.

Case 1: $1 \leqslant l \leqslant k$. We have

$$
\sum_{i=1}^{l}(x \otimes z)_{i}^{\downarrow}=\left(\alpha+\frac{1}{2} \beta\right) \sum_{i=1}^{l / 2} z_{i},
$$

while

$$
\sum_{i=1}^{l}(y \otimes z)_{i}^{\downarrow}=\alpha \sum_{i=1}^{l} z_{i}
$$

Thus

$$
\begin{aligned}
\sum_{i=1}^{l}(y \otimes z)_{i}^{\downarrow}-\sum_{i=1}^{l}(x \otimes z)_{i}^{\downarrow} & =\alpha \sum_{i=l / 2+1}^{l} z_{i}-\frac{1}{2} \beta \sum_{i=1}^{l / 2} z_{i} \\
& =\sum_{i=1}^{l / 2}\left(\alpha z_{l / 2+i}-\frac{1}{2} \beta z_{i}\right) .
\end{aligned}
$$

This last quantity is a sum of positive terms (by the definition of $\alpha$ and $\beta$ ), so the inequality (5) is strict.

Case 2: $k+1 \leqslant l<2 k$. We have

$$
\sum_{i=1}^{l}(x \otimes z)_{i}^{\downarrow}=\left(\alpha+\frac{1}{2} \beta\right) \sum_{i=1}^{l / 2} z_{i}
$$

and

$$
\sum_{i=1}^{l}(y \otimes z)_{i}^{\downarrow} \geqslant \alpha+\frac{1}{2} \beta \sum_{i=1}^{l-k} z_{i}
$$

The difference thus satisfies

$$
\sum_{i=1}^{l}(y \otimes z)_{i}^{\downarrow}-\sum_{i=1}^{l}(x \otimes z)_{i}^{\downarrow} \geqslant \alpha \sum_{i=l / 2+1}^{k} z_{i}-\frac{1}{2} \beta \sum_{i=l-k+1}^{l / 2} z_{i} .
$$

Note that the sums on the right-hand side each contain $k$ $-l / 2$ terms. Since $\alpha z_{i}>\frac{1}{2} \beta z_{j}$ for any $i, j$, the difference is positive, and again Eq. (5) holds.

Case 3: $l=2 k$. In this case

$$
\sum_{i=1}^{l}(x \otimes z)_{i}^{\downarrow}=\alpha+\frac{1}{2} \beta
$$

and

$$
\begin{aligned}
\sum_{i=1}^{l}(y \otimes z)_{i}^{\downarrow} & \geqslant \alpha+\frac{1}{2} \beta \sum_{i=1}^{k-1} z_{i}+\frac{1}{2} \beta z_{1} \\
& =\alpha+\frac{1}{2} \beta+\frac{1}{2} \beta\left(z_{1}-z_{k}\right)>\alpha+\frac{1}{2} \beta
\end{aligned}
$$

so the inequality (5) is strict.

Case 4: $2 k+1 \leqslant l \leqslant 3 k$. We have

$$
\sum_{i=1}^{l}(x \otimes z)_{i}^{\downarrow}=\alpha+\frac{1}{2} \beta+\frac{1}{2} \beta \sum_{i=1}^{l / 2-k} z_{i}
$$

while

$$
\sum_{i=1}^{l}(y \otimes z)_{i}^{\downarrow} \geqslant \alpha+\frac{1}{2} \beta+\frac{1}{2} \beta \sum_{i=1}^{l-2 k} z_{i}
$$

The second quantity is clearly larger, so the inequality (5) is strict.

Case 5: $3 k+1 \leqslant l<4 k$. This case is trivial because the sum for $y \otimes z$ is 1 (because there are no more nonzero terms to be added) and the sum for $x \otimes z$ is less than 1 .

We have shown that Eq. (5) holds when $l$ is even (and in the proper range). Now suppose $l$ is odd. From the even cases, it is easily verified that

$$
\sum_{i=1}^{l-1}(x \otimes z)_{i}^{\downarrow}+\sum_{i=1}^{l+1}(x \otimes z)_{i}^{\downarrow}<\sum_{i=1}^{l-1}(y \otimes z)_{i}^{\downarrow}+\sum_{i=1}^{l+1}(y \otimes z)_{i}^{\downarrow}
$$

when $l \in\{1,3, \ldots, 4 k-1\}$. Based on the fact that the components of $(y \otimes z)^{\downarrow}$ are nonincreasing, $\sum_{i=1}^{l}(y \otimes z)_{i}^{\downarrow}$ is greater than or equal to the average of the two sums on the righthand side of Eq. (6). However, $\sum_{i=1}^{l}(x \otimes z)_{i}^{\downarrow}$ is equal to the average of the sums on the left-hand side of Eq. (6), since the components of $(x \otimes z)^{\downarrow}$ appear in pairs. We therefore see that Eq. (5) holds when $l$ is odd. 
Thus, the majorization inequalities are strict for all $l$ between 1 and $4 k-1$ inclusive, so for sufficiently small $\epsilon$, $\left(x_{1}+\epsilon, x_{2}+\epsilon, x_{3}-\epsilon, x_{4}-\epsilon\right) \otimes z<y \otimes z$. However, $\left(x_{1}+\epsilon, x_{2}\right.$ $\left.+\epsilon, x_{3}-\epsilon, x_{4}-\epsilon\right) \nless y$, so our theorem is proved.

\section{CONCLUSION}

While the majorization relation is a fairly well-studied subject, tensor-product induced majorization (i.e., the trumping relation) is an extension of this relation about which comparatively little is known. Trumping is a relatively new notion that allows us to categorize which transformations of entangled states are possible using only local operations and classical communication. Unfortunately, given $x$ and $y$, it is not easy to determine whether $x$ is trumped by $y$. And given $y$, there is no known geometric or function-theoretic categorization of $T(y)$, the set of vectors trumped by $y$; this is in contrast to the case with the majorization relation, where such characterizations do exist. In this paper we have derived a number of results about the trumping relation, in an effort to improve our understanding of this relation.

Recent work has demonstrated additional applications for majorization in quantum information theory [11-13]. For instance, a majorization condition has been shown necessary for a state to be separable [11], and it has also been shown that various majorization conditions must be satisfied by quantum systems undergoing mixing and measurement processes [12]. As discoveries relating majorization to quantum information science are made, new applications for the trumping relation may arise.

\section{ACKNOWLEDGMENTS}

The authors thank Michael Nielsen for introducing us to this subject, providing encouragement and feedback on our results, and generously commenting on the manuscript. We also thank David Beckman for helpful discussions.
[1] C. H. Bennett and G. Brassard, Proceedings of IEEE International Conference on Computers, Systems, and Signal Processing, Bangalore, India, 1984 (unpublished), pp. 175-179.

[2] C. H. Bennett and G. Brassard, IBM Tech. Discl. Bull. 28, 3153 (1985).

[3] C. H. Bennett, G. Brassard, C. Crepeau, R. Josza, A. Peres, and W. K. Wooters, Phys. Rev. Lett. 70, 1895 (1993).

[4] C. H. Bennett and S. J. Wiesner, Phys. Rev. Lett. 69, 2881 (1992).

[5] M. A. Nielsen and I. L. Chuang, Quantum Computation and Quantum Information (Cambridge University Press, Cambridge, 2000).

[6] J. Preskill, http://www.theory.caltech.edu/people/preskill/ ph229/\#lecture
[7] M. A. Nielsen, Phys. Rev. Lett. 83, 436 (1999); note: arXiv e-print quant-ph/9811053.

[8] A. W. Marshall and I. Olkin, Inequalities: Theory of Majorization and Its Applications (Academic, New York, 1979).

[9] M. A. Nielsen, http://www.physics.uq.edu.au/people/nielsen/ info/maj.ps

[10] D. Jonathan and M. B. Plenio, Phys. Rev. Lett. 83, 3566 (1999); note: arXiv e-print quant-ph/9905071.

[11] M. A. Nielsen and J. Kempe, Phys. Rev. Lett. 86, 5184 (2000); note: arXiv e-print quant-ph/0011117.

[12] M. A. Nielsen, Phys. Rev. A 63, 022114 (2001); note: arXiv e-print quant-ph/0008073.

[13] M. A. Nielsen, Phys. Rev. A 62, 052308 (2000); note: arXiv e-print quant-ph/9909020. 